\title{
Reducing medication has become a privilege of wealth in western settings
}

\author{
DAVID HEALY
}

\begin{abstract}
In 20th century healthcare in affluent settings, generating awareness of the hazards of medication followed a rotten apple script. Find patients with a problem, find the documents that a drug company knew about the problem in private but denied it in public, mention the ghost-writing that concealed the problem and lack of access to trial data, perhaps using a clinician or an "insider" to provide dramatic focus and expose the company to media or legal questioning. The sight of a rotten apple being thrown out of the barrel reassured the public that healthcare was now more ethical than before, and gave bioethicists a case example to use in teaching. But what if we have a whole rotten barrel as outlined in this case study? What are the ethics then?
\end{abstract}

Keywords: Affluent health systems, sustainable treatment, mental health referrals, adverse medication effects

\section{A "good" clinic}

Between February 2020 and February 2021, David Healy (1) was one of four psychiatrists taking mental health referrals from 80 primary care physicians working in Guelph, a university city in Ontario, Canada. The practice was typical of all public healthcare systems in affluent countries and affluent practice everywhere.

The most common referrals were for people who were anxious or depressed, $70 \%$ of whom were women, who had been on antidepressants for a decade or more, some of whom had started in their late teens. In some cases, these had been supplemented with other drugs.

Another group of patients wondered if they had depression, Attention Deficit Hyperactivity Disorder (ADHD) or Autistic Spectrum Disorder (ASD). This group included a number of women and men who could instead be described as extraverted or introverted in temperament rather than ill. Extraverts complain of lack of focus; introverts complain

Author: David Healy (David.Healy54@gmail.com), Professor of Psychiatry, Department of Family Medicine, McMaster University, Hamilton, CANADA

To cite: Healy D. Reducing medication has become a privilege of wealth in western settings. Indian J Med Ethics. Published online first on November 4, 2021. DOI: 10.20529/IJME.2021.084.

Peer reviewer: An anonymous reviewer

c Indian Journal of Medical Ethics 2021 about too much focus. Extraverts take risks and are impulsive, introverts manage risks and ruminate. Extraverts find the features of ADHD described in the lay media fit them perfectly. Many university students engineer an ADHD diagnosis, believing a stimulant will help their academic work (2). Extraversion and introversion are biologically based elements that shape personalities. Medication can help but is not a cure, and recently, the treatment of ADHD by stimulants has been linked to an earlier onset of Parkinson's disease (3).

A third group were people under work stress linked to changes in the management culture in factories, universities and healthcare settings in the last decade. These physician referrals asked if the person is depressed or anxious conditions that locate the problem in the person. A neutral option is burnout or adjustment disorder, which tread the line between securing a disability payment and not locating the problem within the individual.

Few referrals had prior admissions for mental illness, even though some had up to six different diagnoses and were taking nine different psychotropic drugs, in addition to other medication.

\section{Mild to moderate "mental illness"}

There is more uncertainty in referrals from primary care physicians than is often appreciated. The superficial dynamic is that these referrals are for minor mental illness and an expert in serious mental illness will find these cases easy. Accepting there is a continuity in some cases, primary care problems are not in general continuous with traditional mental illnesses such as schizophrenia or manic-depressive illness.

Severe mental illnesses are relatively easy to diagnose, and the treatment options are more straightforward than many primary care cases. Primary care throws up a lot of anxiety and distress stemming from existential or social issues, as well as conditions where the appropriate response is diagnostic and therapeutic uncertainty. Some cases involve medically unexplained symptoms (MUS), which it can be a mistake to view as "mental".

Aside from a small number of cases that may evolve into mental illness in need of secondary care or have neuropsychiatric features that specialist input can help with, or may require a turn to off-label therapeutic options, a 
primary care physician will usually know the person, their family and circumstances, better than a psychiatrist could on the basis of a single meeting, and without contact with family members or the ability to see changes over time.

There is, however, pressure on family doctors to do what specialists recommend.

\section{SSRI burnout}

Some of the main treatments for primary care nervous problems, like the selective serotonin reuptake inhibitors (SSRIs), are now 30 years old. While their use has largely been proper and helpful, professional bodies like Britain's Royal College of Psychiatrists now accept that these drugs can cause dependence and can be difficult to stop (4). In practice, this means some of the nervous problems patients bring to their family physician will be breakthrough anxiety and depressive symptoms for which no treatments are mandated. This is a confronting situation for all concerned.

Each family doctor may have only a few cases like this, but where several doctors refer to the same psychiatrist, s/he may end with a substantial proportion of cases falling under this heading and no easy solution to offer. Switching to other antidepressants or adding an adjuvant is not a reliable answer and often causes further problems.

It can be difficult to offer a clear management plan. The patient's agreement to any plan is important especially if they are likely to be faced with withdrawal difficulties. Opening up a space, therefore, where the patient, supported by a family doctor, can disagree with the "expert" is important in these cases.

\section{The family doctor-centred approach}

Family doctors often complain that specialists rarely consult them on a case. With every referral, Healy messaged family doctors to let them know there was an entry in the medical record. These entries often called for family doctor input. In tricky cases, the messages made clear Healy's willingness to engage further and gave his phone number.

Some doctors phoned. In phone calls, they were told Healy was there to support them, even if they opted for a different course of treatment to the one he suggested.

Many messaged back thanking him for the entry to the record, and in some cases explicitly agreeing where, for instance, he had not supported an ADHD diagnosis.

Nevertheless, some family doctors may have been concerned that a willingness to recognise adverse effects and the fact that prior treatments may have painted the patient into a corner, that he might not support them should there be a complaint.

There is a difficult area here between the pragmatic "Give us a view that will help us move this case forward", and medicolegal concerns such as "Give us a view that has a basis in guidelines that will give us cover, should things go wrong."

\section{The bureaucratic or person-centred approach}

Family physicians globally say they do not read reports from psychiatrists other than the closing diagnosis and treatment. The only person with an interest in reading everything is the person referred.

For over 30 years in Britain, Healy provided all outpatients with copies of all reports on them. These letters were framed with the person in mind rather than their doctor. They were light on medical jargon and where possible, used the person's own words. No doctor in 30 years complained about the letters going to the person referred, and they were appreciated by patients $(5,6,7)$.

In Guelph, having notified colleagues of this practice and received support, Healy adopted the same practice, in this case emailing the entry in the electronic medical record (EMR) to the person referred. The reports supported the courses of action initiated by family doctors, even where these had stopped working. Healy made clear he would have taken the same approach in the first instance. Having asked about the relationship between patient and referring doctor, he phrased the report accordingly. The report invited patients to research things that had arisen in the interview including possible drug options, and to check these and other issues with their family doctor.

During this period, the services were operating in lockdown, which meant phone or video supplemented in-person consults. Arranging follow-up appointments with other colleagues could take weeks, which was a problem for anyone having difficulties with change in medication.

Healy's patients had their reports half an hour after a consult and were able to email him any time of any day of the week if they had problems, and could be seen within days. The normal bureaucratic wall between people and their doctors was not there.

\section{Medical consumerism}

Working two days a week, over a year Healy had seen 300 separate referrals. Aware that what was being offered was novel, he proposed a quality improvement project aimed at assessing what physicians and patients made of this new way of working.

Instead, in February 2021, at a meeting he was informed about only 15 minutes beforehand, his input to the Guelph family health team was terminated. An undetermined number of doctors and/or patients, he was told, had been unhappy at an apparently insufficient offering of further diagnoses and treatments. There was no complaint of malpractice or investigation of any incident, and a separate clinic at which he was working was happy for him to continue.

The Guelph system claimed not to be able to stream patients 
allowing those who want an approach aimed at sustainable treatment with some sensitivity to the possibility that current treatments may be having adverse effects, to have it. The system, he was told, must have a standard approach, and at present at least some doctors and patients expect more diagnoses and treatments. The default is to medical consumerism and an adherence to guidelines for diseases rather than guidelines for people, one criterion of which in the light of current evidence might be that no-one should be on more than five drugs (8).

Despite growing discussion about deprescribing in university departments and among clinicians, in addition to patients interested in exploring this option, there is at present nowhere in Canada that offers sustainable treatment, ie treatment that minimises drug use, as part of its mix.

Where once access to treatment was a privilege of wealth, public health systems are now geared toward increasing access to medicines. In western medical systems at least, being able to reduce that resulting medication burden appears to have become a privilege of wealth.

In North America, referrals increasingly come from people approaching a family doctor claiming they have ADHD, ASD, or whatever, expecting to be referred to a specialist who will confirm the diagnosis and treatment.

These diagnoses are fashionable and can suck people in, young people especially, in the way cults do (9: ch 10). While some doctors will try to persuade patients that consumerism and poisons (medicines) or mutilations (surgery) are not natural bedfellows, and while some patients can be persuaded, an increasing number of them complain if they are not given what they want."We have the rating scale score, where's our drug?"

There is a case for saying what a person demands, a person should get, and in the case of terminations of pregnancy, gender reassignment and medical assistance in dying, Ontario courts have said that doctors unwilling to support these options must make an effective referral or consider switching to a non-medical career.

A standardised medical consumerism poses a risk to patients. If we standardise processes to the point where everyone gets the diagnosis they want, based on rating scales they selfadminister, we will end up with diagnostic and prescribing cascades, evident in Guelph primary care patients - as demonstrated in the following example:

A brief upswing of mood diagnosed as bipolar disorder will lead to a mood-stabilizer, ultimately several, one of which will be an antipsychotic. When asked in clinic how they are, any mention of loss of focus, which antipsychotics are designed to produce, risks leading to an ADHD scale and diagnosis and prescription of a stimulant. A dopamine agonist is now chasing a dopamine antagonist, and both can produce depressive symptoms, leading to further antidepressants.

A standardised medical consumerism hinges on the idea that medicines are sacraments (can do no harm). On this basis, the managers who increasingly run health services are turning to cheaper prescribers such as nurses and pharmacists. We need to consider whether we have reached a point where professional discretion is no longer valuable.

\section{Pandemic}

The turn to virtual consults as a result of the Covid-19 pandemic may aggravate these risks. Gone is the feel for someone else from a look or body language that may convey a message at odds with a patient's words, especially when treatment is going wrong. This is a good reason for increased liaison between a specialist and a primary care physician who is more likely to have laid eyes on the person before and to know how much their demeanour and deportment now differs.

The switch to new ways of working triggered by the pandemic has already led to more prepping of patients with questionnaires that constrain later clinical interactions and diagnostic possibilities. An increasing number of clinical evaluations and treatment recommendations come from out-of-state doctors, often through phone apps.

Our oversight of who is delivering care and the conversations between family doctors and patients, and between family doctors and mental health personnel, necessary to good care are slipping away.

\section{Rotten barrel?}

This state of affairs would have been inconceivable a few years ago. It stems directly from a ghost-writing of the medical literature concerning on-patent drugs and total sequestration of clinical trial data. This change has confirmed the medicines in common use as sacraments, rather than the poisons they were once viewed as. The magic of medicine once lay in the doctor who brought good out of the use of a poison, but the magic now lies in pills, and doctors risk being swept away in the rush to get sacraments (9: ch 15).

The traditional script for rooting out unethical practice will only make this problem worse by reassuring both doctors and patients that a problem has been found and the barrel minus a rotten apple is now safer than it was. We have a systemic problem with no clear solutions. This account may offer ethicists a case example to use in teaching.

\section{References}

1. Healy D. Conflicting interests in Toronto. Anatomy of a controversy at the interface of academia and industry. Perspect Biol Med. 2002 Spring; 45(2): 250-63.

2. Schwarz A. ADHD Nation: Children, Doctors, Big Pharma, and the Making of an American Epidemic. New York: Scribner; 2016.

3. Baumeister AA. Is Attention-Deficit/Hyperactivity Disorder a risk syndrome for Parkinson's disease? Harvard Rev Psychiatry 2021: 29; 142-58.

4. Press release. The Royal College of Psychiatrists updates information on stopping antidepressants. 2020 Sep 23[cited 2021 May 4] 
Available from: https://www.rcpsych.ac.uk/news-and-features/latestnews/detail/2020/09/23/new-information-on-stoppingantidepressants

5. Fitzgerald K, Williams B, Healy D. Shared care? Some effects of patient access to medical communications. J Mental Health 1997: 6; 37-46.

6. Healy D. Involving users in mental health services in the era of the word processor and the database. In Crosby C, Barry M (eds.). Community Care: Evaluation of the Provision of Mental Health Services
1995, Aldershot: Avebury Press: 1995, 209 - 231.

7. Williams B, Healy D. Perceptions of illness causation among new referrals to a community mental health team: "explanatory model" or "exploratory map". Soc Sci Med 2001:53; 465-76.

8. Garfinkel D, Mangin D. Feasibility study of a systematic approach for discontinuation of multiple medications in older adults. Arch Intern Med. 2010: 170; 1648-54.

9. Healy D. Shipwreck of the Singular. Healthcare's Castaways. Toronto, Samizdat-Health. 2021. 